\title{
Farnesylation Process
}

National Cancer Institute

\section{Source}

National Cancer Institute. Farnesylation Process. NCI Thesaurus. Code C41582.

A likely membrane attachment device, farnesylation involves enzymatic covalent addition of farnesyl group(s) (three isoprene units) to polypeptide chains that contain the carboxyl terminal motif CAAX. The addition involves cleavage and addition to the free thiol group of cysteine moiety, and methylation of the free carboxylate group on the protein's carboxyl terminus. 